\title{
Hematopoietic progenitors passing through early embryonic CD4-positive stage are long-lived and give rise to myeloid and lymphoid progeny.
}

Apostol K. Apostolov ${ }^{1}$ and Julien C. Marie ${ }^{1 *}$

${ }^{1}$ Tumor Escape Resistance and Immunity department, Cancer Research Center of Lyon INSERM U1052, CNRS UMR 5286, Centre Léon Bérard, Claude Bernard Université Lyon 1, 69373, Lyon, France

* To whom correspondence should be addressed

Julien C. Marie: (julien.marie@inserm.fr)

This manuscript has been withdrawn as it was submitted and made public without the full consent of all the authors. Therefore, the authors do not wish this work to be cited as reference for the project. If you have any questions, please contact the corresponding author. 